Article

\title{
Performance of Thyme Oil@Na-Montmorillonite and Thyme Oil@Organo-Modified Montmorillonite Nanostructures on the Development of Melt-Extruded Poly-L-lactic Acid Antioxidant Active Packaging Films
}

\author{
Aris E. Giannakas ${ }^{1, *(\mathbb{D}}$, Constantinos E. Salmas ${ }^{2, * \mathbb{D}}$, Areti Leontiou ${ }^{3} \mathbb{D}$, Dimitrios Moschovas ${ }^{2}$, Maria Baikousi ${ }^{2}$, \\ Eleni Kollia 4(D), Vasiliki Tsigkou 4 (D), Anastasios Karakassides ${ }^{2}$, Apostolos Avgeropoulos ${ }^{2}$ (D) \\ and Charalampos Proestos $4, * \mathbb{D}$ \\ check for \\ updates \\ 1 Department of Food Science and Technology, University of Patras, 30100 Agrinio, Greece \\ 2 Department of Material Science \& Engineering, University of Ioannina, 45110 Ioannina, Greece; \\ dmoschov@uoi.gr (D.M.); mbaikou@uoi.gr (M.B.); tasos.karakassides@gmail.com (A.K.); \\ aavger@uoi.gr (A.A.) \\ 3 Laboratory of Food Technology, Department of Business Administration of Agricultural and Food Enterprises, \\ University of Patras, 30100 Agrinio, Greece; aleontiu@upatras.gr \\ 4 Laboratory of Food Chemistry, Department of Chemistry, National and Kapodistrian University of Athens \\ Zografou, 15771 Athens, Greece; elenikollia@chem.uoa.gr (E.K.); vtsigkou@chem.uoa.gr (V.T.) \\ * Correspondence: agiannakas@upatras.gr (A.E.G.); ksalmas@uoi.gr (C.E.S.); harpro@chem.uoa.gr (C.P.)
}

Citation: Giannakas, A.E.; Salmas, C.E.; Leontiou, A.; Moschovas, D.; Baikousi, M.; Kollia, E.; Tsigkou, V.; Karakassides, A.; Avgeropoulos, A.; Proestos, C. Performance of Thyme Oil@Na-Montmorillonite and Thyme Oil@Organo-Modified

Montmorillonite Nanostructures on the Development of Melt-Extruded Poly-L-lactic Acid Antioxidant Active Packaging Films. Molecules 2022, 27, 1231. https://doi.org/10.3390/ molecules27041231

Academic Editors: Carmen Chifiriuc and Eliza Oprea

Received: 19 January 2022

Accepted: 9 February 2022

Published: 11 February 2022

Publisher's Note: MDPI stays neutral with regard to jurisdictional claims in published maps and institutional affiliations.

Copyright: (c) 2022 by the authors. Licensee MDPI, Basel, Switzerland. This article is an open access article distributed under the terms and conditions of the Creative Commons Attribution (CC BY) license (https:/ / creativecommons.org/licenses/by/ $4.0 /)$.

\begin{abstract}
Today, the use of natural biodegradable materials in the production processes is more and more adopted by industry to achieve cyclic economy targets and to improve environmental and human health indexes. Active packaging is the latest trend for food preservation. In this work, nanostructures were prepared by incorporation of thyme oil with natural natrium-montmorillonite and organo-montmorillonite with two different techniques, direct impregnation and the green evaporation-adsorption process. Such nanostructures were mixed with poly-L-lactic-acid for the first time via an extrusion molding process to develop a new packaging film. Comparisons of morphological, mechanical, and other basic properties for food packaging were carried out via XRD, FTIR, TG, SEM/EDS, oxygen and water vapor permeation, and antimicrobial and antioxidant activity for the first time. Results showed that poly-L-lactic-acid could be modified with clays and essential oils to produce improved active packaging films. The final product exhibits food odor prevention characteristics and shelf-life extension capabilities, and it could be used for active packaging. The films based on OrgMt clay seems to be more promising, while the thyme oil addition improves their behavior as active packaging. The PLLA/3\%TO@OrgMt and PLLA/5\%TO@OrgMt films were qualified between the tested samples as the most promising materials for this purpose.
\end{abstract}

Keywords: thyme oil; sodium montmorillonite; poly-L-lactic acid; active packaging; antioxidant activity; antimicrobial activity

\section{Introduction}

Today, green and circular economies favor the development of nanocomposite packaging materials from renewable source-based biodegradable plastics or biopolymers [1] Polylactides or poly(lactic acid) (PLA) is one of the most promising materials between the renewable source-based biodegradable plastics since it is thermoplastic, biodegradable, and biocompatible and exhibits high-strength, high-modulus, and good processability [2-5]. PLA is a linear aliphatic thermoplastic polyester produced either by the polycondensation of lactic acid or by the ring-opening polymerization of lactide. Lactide is a cyclic dimer prepared by the controlled depolymerization of lactic acid, which is obtained from the fermentation of renewable sugar feedstock, such as corn or sugar beets. Polymerization of 
a racemic of L-lactides leads to the synthesis of poly-L-lactide (PLLA). Since production cost has been lowered by new technologies and large-scale production, the application of PLA has been extended to materials for packaging applications [3-6].

Furthermore, one of the most recent applications of nanotechnology [7] in the food industry and food packaging sector is the development of polymer nanocomposite-based packaging films [8,9]. Among the nanomaterials used for polymer-based nanocomposites, clay-type nanomaterials are the most frequently used because they are naturally abundant low cost materials and because of the improved mechanical and barrier properties they add to the obtained polymer/clay nanocomposites [10]. More recently, clay-type nanomaterials have been proposed as an ideal nanocarrier for bioactive compounds, e.g., essential oils, for active food packaging films [8,11-13]. Such nanomaterials exhibit controlled released antimicrobial activity, antioxidant activity, and aroma properties. The obtained essential oils/nanoclay hybrids [14] can be added into polymer or biopolymer matrixes, providing active packaging films and/or coatings with potential controllable antioxidant and/or antimicrobial activity [15]. The term active packaging refers to packaging technologies which are used with foods, pharmaceuticals, and other products. The aim of the use of such technologies is to extend products shelf-life, to monitor their freshness and quality, and to increase their safety and accessibility by consumers [16].

Rhim et al. [17] prepared via a solution casting method PLA-based nanocomposite films with different types of nanoclays, such as commercial Cloisite $\mathrm{Na}^{+}$, Cloisite 30B, and Cloisite 20A. Cloisite 20A was the most effective to improve the water vapor barrier property and Cloisite 30B to improve the antibacterial properties. Recently, Ramos et al. [18] prepared PLA/thymol/nanoclay nanocomposite film via a melt-extruded method. They added $2.5 \mathrm{wt} \%$ and $5 \mathrm{wt} \%$ of a commercial nanoclay with code name Dellite ${ }^{\circledR} 43 \mathrm{~B}$ (D43B) and $8 \mathrm{wt} \%$ of thymol in PLA. The results from this study indicated that the diffusion of thymol through the PLA matrix was influenced by the presence of the nanoclay. Villegas et al. [6] prepared intercalated PLA/organoclay/nanocomposites film via melt extrusion and impregnated with thymol and cinnamaldehyde through supercritical impregnation using carbon dioxide.

Recently, PLA was incorporated with Na-montmorillonite (NaMt) and commercial organomodified montmorillonite (OrgMt) via two methods, the direct method and the masterbatch method to produce active packaging films. Comparison and evaluation of the different materials and the different producing processes were carried out via mechanical properties and structure characterization measurements and the results reported in [19]. In this work a direct impregnation and a novel green evaporation-adsorption method were used to modify Na-montmorillonite (NaMt) and commercial organomodified montmorillonite (OrgMt) with an essential oil, thyme oil (TO), to produce nanostructures similar to others reported in [20]. In the past, these obtained TO@NaMt and TO@OrgMt hybrid nanostructures were connected successfully with chitosan [21], Low-Density Polyethylene [12], and polystyrene [22] matrixes. The novelty of this work is that these new nanohybrid materials, i.e., the TO@NaMt and the TO@OrgMt, which were prepared via a classic method and via a novel evaporation-adsorption process, were used to develop novel poly-L-Lactic acid (PLLA)/thyme oil/montmorillonite nanocomposite active films via an extrusion molding process. The obtained PLLA/TO@NaMt and PLLA/TO@OrgMt films were characterized by XRD analysis, FTIR spectroscopy, SEM images, and TG experiments. Moreover, to evaluate the potential use of such active films in the food packaging industry, tensile properties, water, and oxygen barrier properties, as well as the antioxidant and antimicrobial activity of all the obtained PLLA/TO@NaMt and PLLA/TO@OrgMt films, were measured.

\section{Results}

2.1. XRD

Table 1 lists the code names of all samples and the used amounts of PLLA, NaMt, OrgMt, TO@NaMt, and TO@OrgMt. 
Table 1. Code names and amounts in grams used for the preparation of pure PLLA film and all obtained PLLA/NaMt, PLLA/TO@NaMt, PLLA/OrgMt, and PLLA/TO@OrgMt nanocomposite films.

\begin{tabular}{cccccc}
\hline Code Name & PLLA & NaMt & OrgMt & TO@NaMt & TO@OrgMt \\
\hline PLLA & 5 & - & - & - & - \\
PLLA/1NaMt & 4.95 & 0.05 & & & - \\
PLLA/3NaMt & 4.85 & 0.15 & - & - & \\
PLLA/5NaMt & 4.75 & 0.25 & & & \\
PLLA/1TO@NaMt & 4.95 & & & 0.05 & - \\
PLLA/3TO@NaMt & 4.85 & - & - & 0.15 & - \\
PLLA/5TO@NaMt & 4.75 & - & - & 0.25 & - \\
PLLA/1OrgMt & 4.95 & & 0.05 & & - \\
PLLA/3OrgMt & 4.85 & - & 0.15 & - & 0.05 \\
PLLA/5OrgMt & 4.75 & & 0.25 & & 0.15 \\
PLLA/1TO@OrgMt & 4.95 & - & & - & 0.25 \\
PLLA/3TO@OrgMt & 4.85 & - & - & - & \\
PLLA/5TO@OrgMt & 4.75 & - & - & & \\
\hline
\end{tabular}

Figure 1 presents the XRD plots of PLLA/NaMt (Figure 1a), PLLA/OrgMt (Figure 1b), PLLA/TO@NaMt (Figure 1c), and PLLA/TO@OrgMt (Figure 1d) nanocomposite films. XRD plots of pure PLLA, NaMt, OrgMt as received, and TO@NaMt and TO@OrgMt nanostructures are also included to compare with the obtained films.

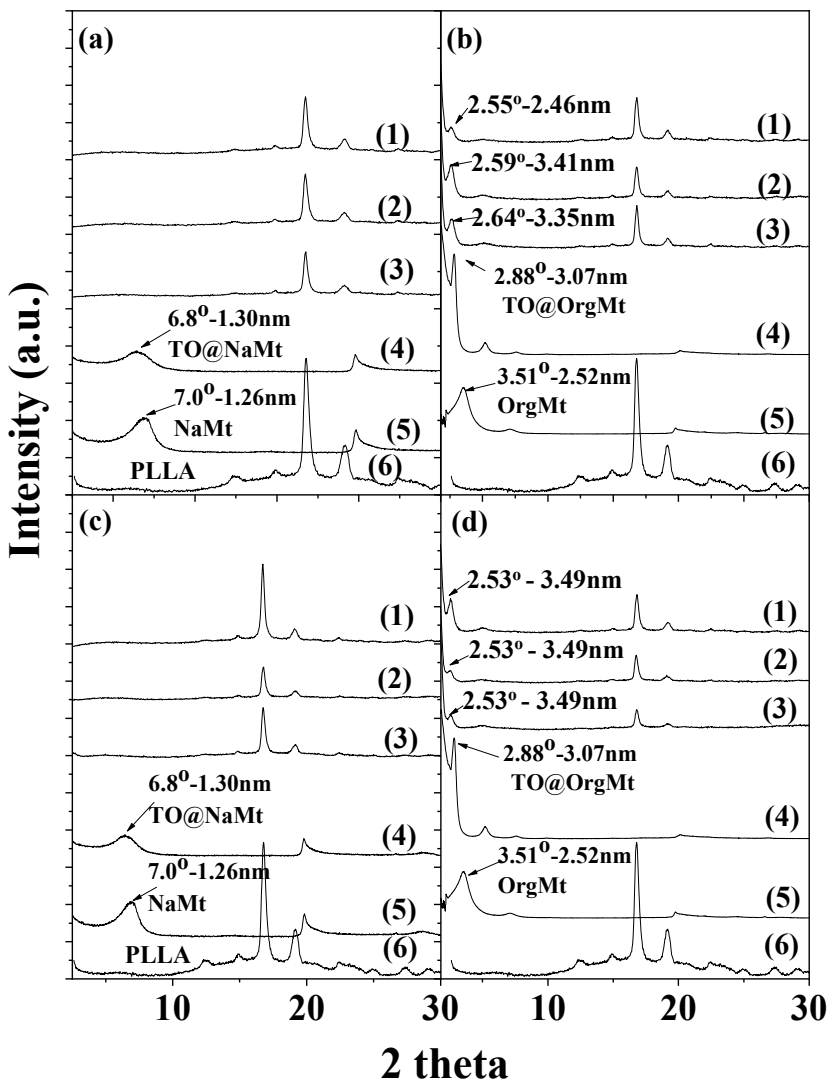

Figure 1. XRD plots of (a) PLLA/1NaMt (1), PLLA/3NaMt (2), PLLA/5NaMt (3), TO@NaMt hybrid nanostructure (4), NaMt as received (5), and PLLA (6); (b) PLLA/1OrgMt (1), PLLA/3OrgMt (2), PLLA/5OrgMt (3), TO@OrgMt hybrid nanostructure (4), OrgMt as received (5), and PLLA (6); (c) PLLA/1TO@NaMt (1), PLLA/3TO@NaMt (2), PLLA/5TO@NaMt (3), TO@NaMt hybrid nanostructure (4) NaMt as received (5), and PLLA (6); (d) PLLA/1TO@OrgMt (1), PLLA/3TO@OrgMt (2), PLLA/5TO@OrgMt (3), TO@OrgMt hybrid nanostructure (4), OrgMt as received (5), and PLLA (6). 
PLLA shows its most intense diffraction peaks at $2 \theta$ values of 16.6 and 18.96 degrees, which is in agreement with previous reports $[23,24]$. The intension of such peaks decreased in all XRD plots of PLLA/NaMt, PLLA/TO@NaMt, PLLA/OrgMt, and PLLA/TO@OrgMT nanocomposites. This fact indicates the interplay between PLLA molecules and nanoclay platelets. The decrease of intense of the characteristic PLLA peaks is higher for OrgMt- and TO@OrgMt-based nanocomposites than for NaMt- and TO@NaMt-based nanocomposites.

It is obvious from Figure 1a,c that the major diffraction peak of NaMt clay is at around 2theta $=7.0^{\circ}$. The TO@NaMt modified nanostructure exhibits a broader peak at 2 theta $=6.8^{\circ}$. According to previous reports [14,20], TO molecules were encapsulated on the external surface of NaMt layers, causing a partial exfoliation of clay platelets. The small shift of 2 theta angle from 7.0 to $6.8^{\circ}$ occurs probably due to the insertion of water molecules in the NaMt's interlayer space. It is also obvious from Figure 1a,c that the blending of both NaMt clay and TO@NaMt nanostructure with PLLA chains resulted to XRD plots with a very broad peak at 2 theta around 4.8 and 5.0 degrees. Thus, it seems that PLLA/NaMt and PLLA/TO@NaMt nanocomposites favored the formation of an exfoliated nanocomposite structure [12].

In Figure $1 b, d$, the basal space of OrgMt clay is observed at 2 theta $=3.50^{\circ}$. TO@OrgMt hybrid nanostructure exhibits a clear shift at 2 theta $=2.88^{\circ}$ degrees. This result is in accordance with previous report [20]. In this report, it was shown [20] that TO molecules encapsulated in the internal space of OrgMt clay platelets. The basal space peak of PLLA/1OrgMt, PLLA/3OrgMt, and PLLA/5OrgMt film was at 2theta, $2.55^{\circ}, 2.59^{\circ}$, and $2.64^{\circ}$, respectively, while the basal space peak of all PLLA/1O@OrgMt, PLLA/3TO@OrgMt, and PLLA/5TO@OrgMt films was at around 2theta $=2.53^{\circ}$. These results indicated the formation of an intercalated nanostructure in all cases, and the boost that TO molecules provided to PLLA chains to insert in the OrgMt clay interlayer space.

\subsection{FTIR}

Figure 2 presents the FTIR spectra of all PLLA/xNaMt, PLLA/xTO@NaMt (Figure 2a), and PLLA/xOrgMt, PLLA/xTO@OrgMt, (Figure 2b) nanocomposite films as well as FTIR spectra of "blank" PLLA film for comparison. In all spectra, the main characteristic peaks of both PLLA are observed. As it is denoted (see dot lines in Figure 2a,b), at $3510 \mathrm{~cm}^{-1}$ the $\mathrm{OH}$ stretch band, at 2941 and $2993 \mathrm{~cm}^{-1}$ the $\mathrm{CH}$ and $\mathrm{CH} 3$ stretching bands, and at $1750 \mathrm{~cm}^{-1}$ the characteristic band of the CO ester group of PLLA chain is observed [25].

In such polymer/clay nanocomposite films, the interplay with clay platelets is indicated by the new bands at 3630,918,660, and $470 \mathrm{~cm}^{-1}$, which are accredited to O-H stretching of structural hydroxyl groups, Al-O stretching, $\mathrm{Mg}-\mathrm{O}$ bond, and Si-O stretching of the tetrahedral silica layers of modified clays, respectively [26,27]. In the case of both NaMt- and OrgMt-based nanocomposites, the bands at 3630, and $470 \mathrm{~cm}^{-1}$ of Al-O and Si-O stretchings are most identified (see dot line in Figure 2a,b) and imply the interplay between clay platelets and PLLA chains. With a more careful look at Figure 2 (see the band at $470 \mathrm{~cm}^{-1}$ ), it is observed a higher interplay for OrgMt-based than NaMt-based samples. In advance, this interplay is higher for TO@NaMt and TO@OrgMt films as compared to NaMt and OrgMt films, correspondingly. This probably occurs due to the hydrophobic nature of the PLLA, which is matched with the hydrophobic nature of the TO. The absence of FTIR peaks, which indicate the existence of TO molecules and its derivatives, implies that such molecules are inside the polymer matrix and not on the surface of it. Moreover, the TO amount is too low compared to that of the PLLA and the clay.

\subsection{TG-DTA}

In Figure 3, the TG plots of pure PLLA, "blank" PLLA/NaMt, and PLLA/OrgMt samples as well as all obtained PLLA/TO@NaMt and PLLA/TO@OrgMt nanocomposite films are presented. For all samples, the degradation step starts at around $300-320{ }^{\circ} \mathrm{C}$ and ends at 350-400 ${ }^{\circ} \mathrm{C}$. 

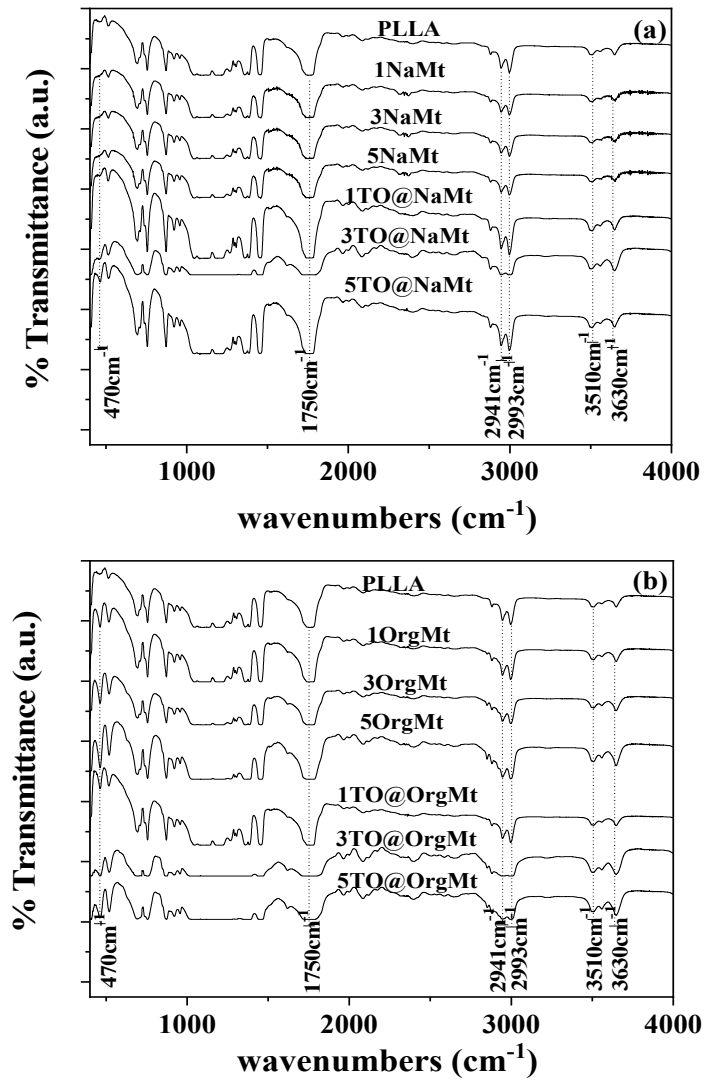

Figure 2. FTIR plots of (a) pure PLLA, PLLA/xNaMt, and PLLA/xTO@NaMt nanocomposites; (b) pure PLLA, PLLA/xOrgMt, and PLLA/xTO@OrgMt nanocomposites.
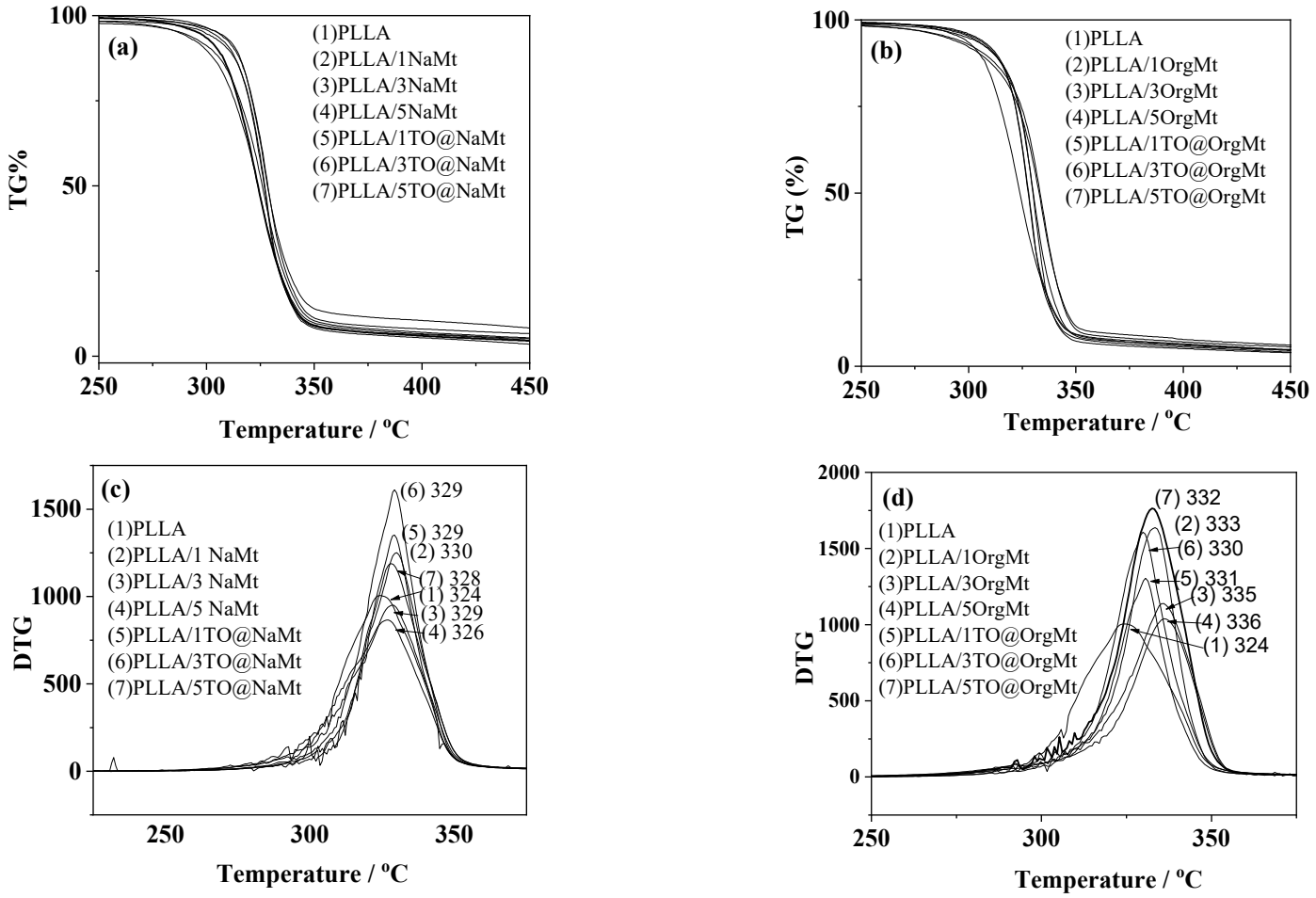

Figure 3. TG plots of (a) pure PLLA, PLLA/xNaMt, and PLLA/xTO@NaMt nanocomposites, and (b) pure PLLA, PLLA/xOrgMt, and PLLA/xTO@OrgMt nanocomposites. DTG plots of (c) pure PLLA, PLLA/xNaMt, and PLLA/xTO@NaMt nanocomposites, and (d) pure PLLA, PLLA/xOrgMt, and PLLA/xTO@OrgMt nanocomposites. 
The temperature value where the degradation process starts is indicative for the thermal strength of such PLLA/nanoclay nanocomposite films. DTG plots for each sample are depicted in Figure 3, and the calculated degradation temperature for each sample is listed. In the case of NaMt-based samples, the addition of extra NaMt caused a slightly increase to the degradation temperature of samples. Moreover, the addition of TO caused a slight decrease to the degradation temperature compared with samples without TO. The addition of extra OrgMt to the OrgMt-based nanocomposites showed a higher increase of the degradation temperature, compared to respective of the NaMt-based samples. The addition of TO caused a slight increase to the degradation temperature of PLLA/1TO@OrgMt as compared to the respective of the PLLA/1OrgMt sample but a slight decrease to the degradation temperature of the PLLA/3TO@OrgMt and PLLA/5TO@OrgMt samples as compared to the respective of the PLLA/3OrgMt and PLLA/5OrgMt samples. The higher increase in thermal stability obtained for OrgMt-based samples as compared to NaMt-based samples is expected following XRD results where intercalated nanocomposite structures were obtained for all OrgMt-based samples, while exfoliated nanocomposite structures were obtained for all NaMt-based samples. The slight decrease of thermal stability of samples containing TO as compared to the samples that do not contain TO is indicative of the plasticization effect caused by the TO addition.

\subsection{Tensile Properties}

Young's modulus as well as tensile strength and elongation at break values of the tested films are tabulated in Table 2. It is obvious that the addition of NaMt in all cases decrease stiffness, strength, and elongation at break values of PLLA. Addition of modified TO@NaMt nanostructure leads to higher stiffness, strength, and elongation at break values of obtained PLLA/TO@NaMt films compared to the relevant PLLA/NaMt films, but only PLLA/3TO@NaMt films exhibit a little higher stiffness, strength, and elongation at brake values compared to the values exhibited by pure PLLA film. In contrast, addition of OrgMt in the PLLA matrix seems to be more effective.

Table 2. Mean values (Standard Deviation) of modulus of elasticity (E), tensile strength $\left(\sigma_{\mathrm{uts}}\right)$, and \% elongation at break $\left(\varepsilon_{\mathrm{b}}\right)$ of all tested PLLA/NaMt, PLLA/TO@NaMt, PLLA/OrgMt, and PLLA/TO@OrgMt films.

\begin{tabular}{cccc}
\hline Code Name & $\begin{array}{c}\text { Tensile E } \\
\text { (St. Dev.) } \\
\text { (MPa) }\end{array}$ & $\begin{array}{c}\boldsymbol{\sigma}_{\text {uts }}(\mathbf{M P a}) \\
\text { (St. Dev.) }\end{array}$ & $\begin{array}{c}\boldsymbol{\varepsilon}_{\mathbf{b}} \mathbf{( \% )} \\
\text { (St. Dev.) }\end{array}$ \\
\hline PLLA & $2891.3(61.9)$ & $33.9(4.9)$ & $1.4(0.2)$ \\
PLLA/1NaMt & $2460.2(232.3)$ & $31.7(5.4)$ & $1.3(0.2)$ \\
PLLA/3NaMt & $2660.5(137.8)$ & $32.5(6.0)$ & $1.3(0.3)$ \\
PLLA/5NaMt & $1823.3(285.4)$ & $22.0(6.3)$ & $1.1(0.1)$ \\
PLLA/1TO@NaMt & $2609.5(137.8)$ & $32.2(6.0)$ & $1.4(0.1)$ \\
PLLA/3TO@NaMt & $2910.7(268.3)$ & $34.5(2.1)$ & $1.4(0.1)$ \\
PLLA/5TO@NaMt & $2811.4(121.7)$ & $33.5(3.7)$ & $1.5(0.3)$ \\
PLLA/1OrgMt & $2315.5(185.2)$ & $29.9(3.8)$ & $1.8(0.3)$ \\
PLLA/3OrgMt & $2965(210.4)$ & $34.2(6.2)$ & $1.5(0.2)$ \\
PLLA/5OrgMt & $2483(235.2)$ & $22.5(5.3)$ & $1.9(0.2)$ \\
PLLA/1TO@OrgMt & $2971(145.8)$ & $38.7(4.2)$ & $1.9(0.1)$ \\
PLLA/3TO@OrgMt & $3277(181.6)$ & $40.3(4.8)$ & $0.9(0.2)$ \\
PLLA/5TO@OrgMt & $2758.5(206.7)$ & $18.2(5.3)$ & \\
\hline
\end{tabular}

Because of this, the PLLA3OrgMt sample showed higher stiffness, strength, and elongation at break values than pure PLLA. Moreover, addition of TO@OrgMt nanostructure leads to the even higher stiffness, strength, and elongation at break values. In other words, it seems that the OrgMt nanostructure exhibits better interplay with the PLLA matrix than the NaMt nanostructure. This result is in accordance with [17]. The addition of TO boosts the dispersion of both NaMt and OrgMt nanostructures in the PLLA matrix. Optimum 
interplay is observed for the PLLA/TO@OrgMt system. The highest stiffness, strength, and elongation at break values were observed for PLLA/3TO@OrgMt active film.

\subsection{SEM/EDS Results}

The surface morphology of the pure PLLA and PLLA/clay nanocomposite films was investigated using a SEM instrument equipped with an EDS detector. EDS mapping analysis from different areas of the studied nanocomposite films was recorded, and the results confirmed that the clays were homogeneously dispersed in the polymer matrix.

The image in Figure 4 shows the expected smooth morphology of the neat polymer, and in Figure 5 the EDS spectra certify the existence of carbon. Surface and relative cross-section images of PLLA/1TO@NaMt, PLLA/3TO@NaMt, and PLLA/5TO@NaMt are presented in Figure 6. It is obvious from Figure 7a,c,e that the increase of clay content in the nanocomposite materials increases the aggregation degree. This fact can be identified by SEM using a secondary electron signal. EDS was then used to map specific elements of the clay nanostructure and identify especially the presence of silicon. As it is observed in Figure 8, clay agglomeration took place, and the increase in clay concentration led to increased silicon signal.
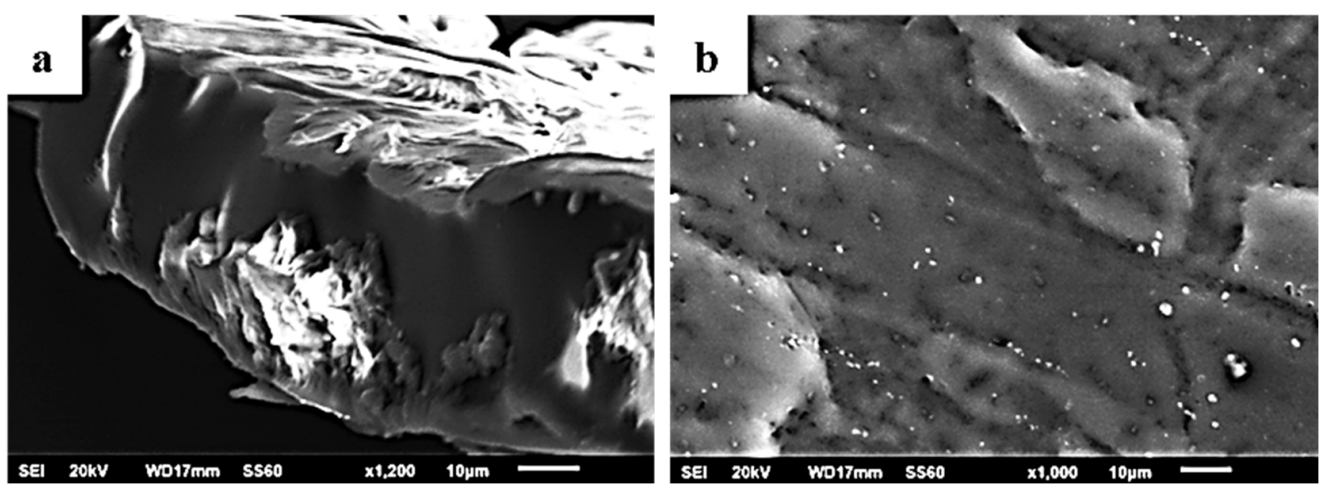

Figure 4. SEM images of the pure PLLA film: (a) cross-section and (b) surface.
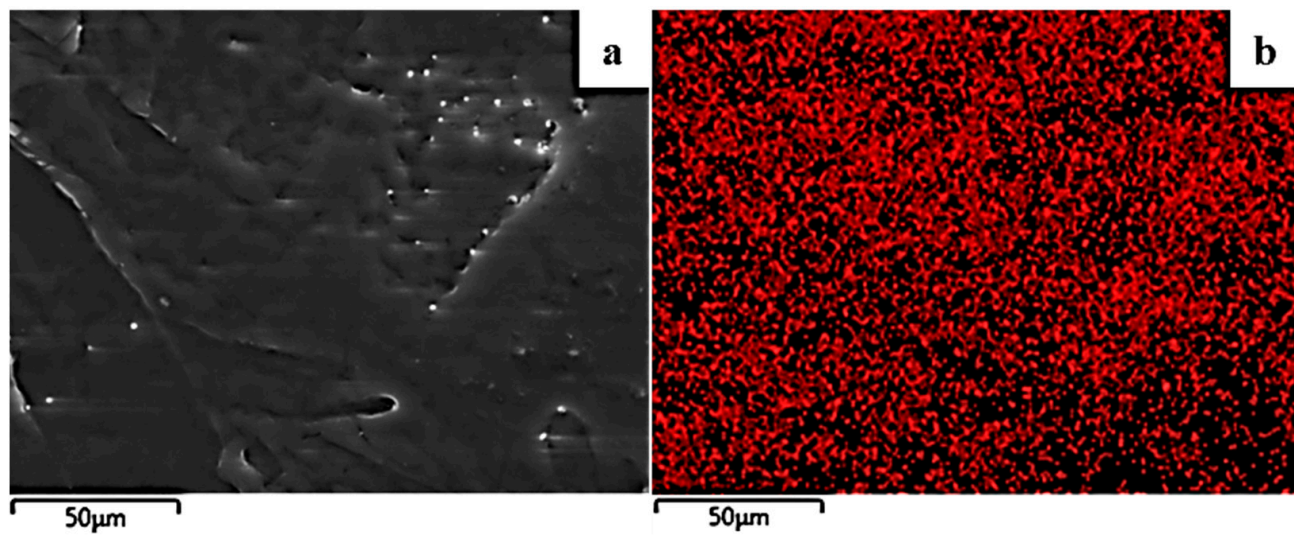

Figure 5. Secondary electron SEM image (a) and respective EDS compositional mapping of carbon (b) for the pure PLLA film. 

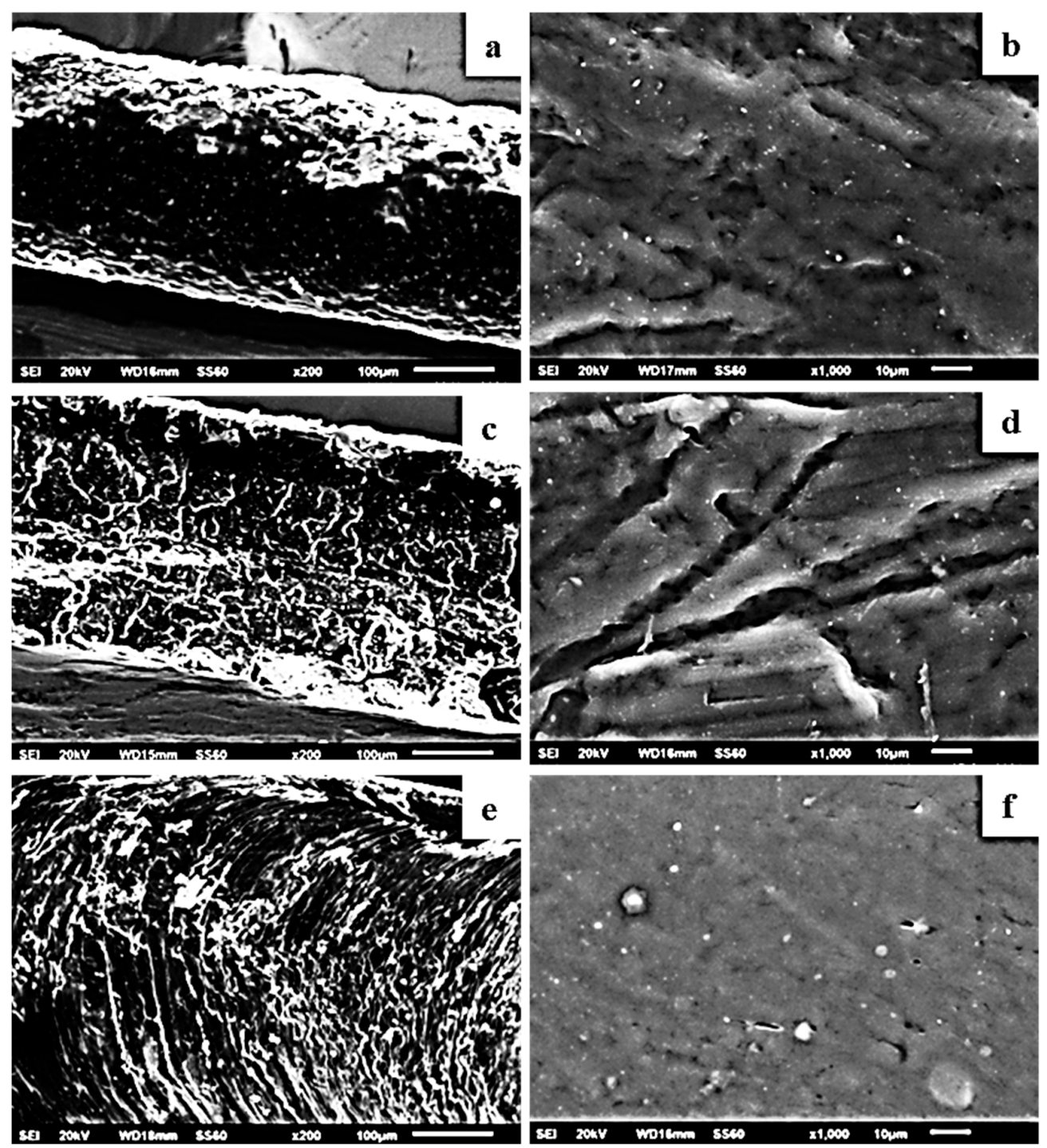

Figure 6. SEM images of cross-section and surface for the films: (a,b) PLLA/1\%TO@NaMt, (c,d) PLLA/3\%TO@NaMt, and (e,f) PLLA/5\%TO@NaMt.

Figure 9 shows the surface $(\mathrm{b}, \mathrm{d}, \mathrm{f})$ and cross-section $(\mathrm{a}, \mathrm{c}, \mathrm{e})$ topology of the PLLA/1TO@OrgMt, PLLA/3TO@OrgMt, and PLLA/5TO@OrgMt nanocomposites, indicating significant clay dispersion within the PLLA polymer matrix. In contrast to the TO@NaMt nanocomposites, the disperse of the TO@OrgMt clays in the polymer matrix led to nonaggregated distributions. This is indicated by the Si signal through EDS mapping. The SEM images and EDS mapping analysis of the PLLA/xTO@OrgMt materials confirm the enhanced compatibility of the TO@OrgMt clay with the PLLA polymer matrix compared to the compatibility of the TO@NaMt clay, which led to rougher PLLA/xTO@NaMt surfaces.

The results from SEM/EDS measurements are in accordance with the XRD results where an intercalated nanocomposite structure for TO@OrgMt-based films and an exfoliated nanocomposite structure for TO@NaMt-based films was confirmed. Both XRD and SEM/EDS results are in total agreement with the higher tensile and thermal strength, which was observed for TO@OrgMt-based films compared to the respective properties of the TO@NaMt-based films. 

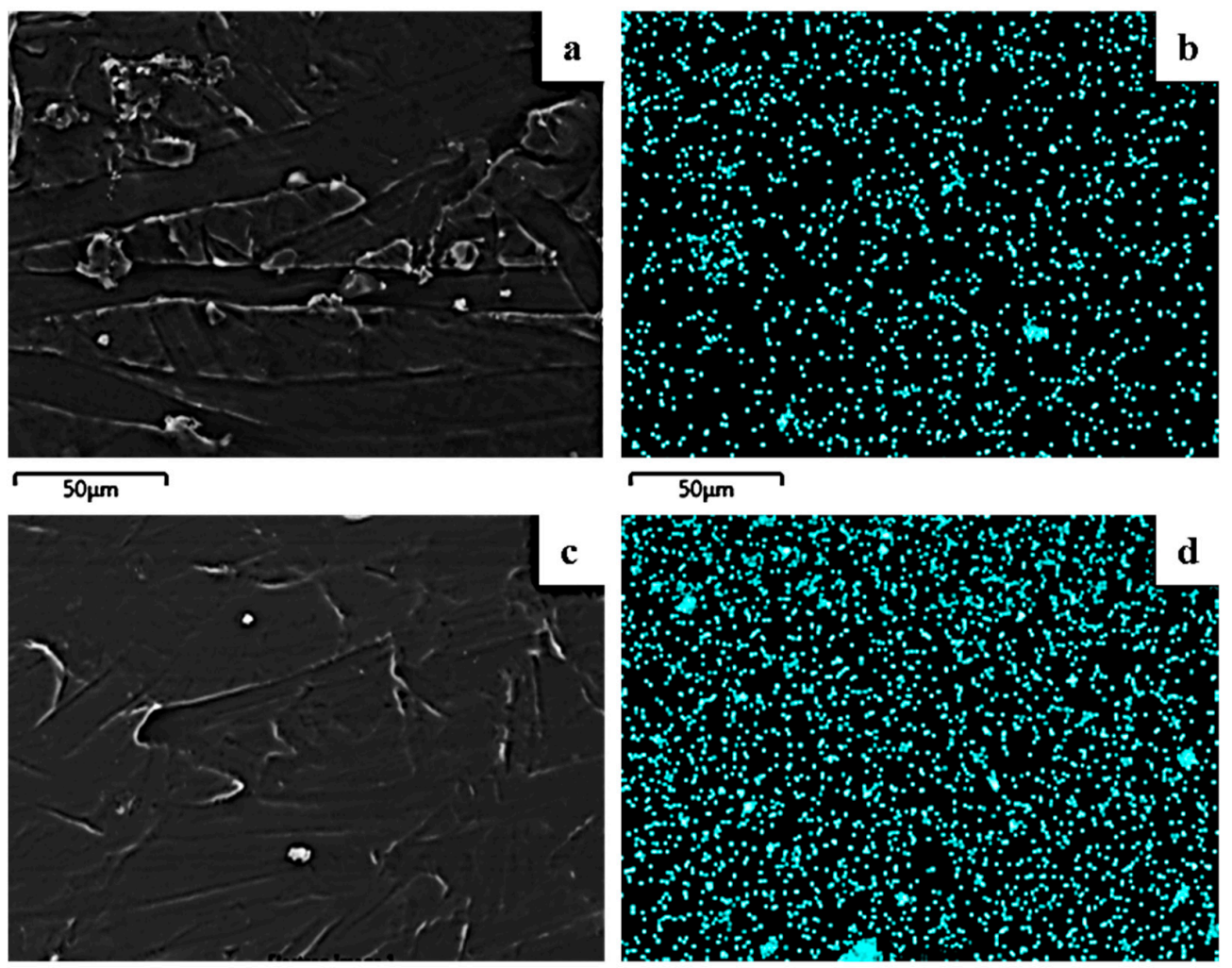

50um
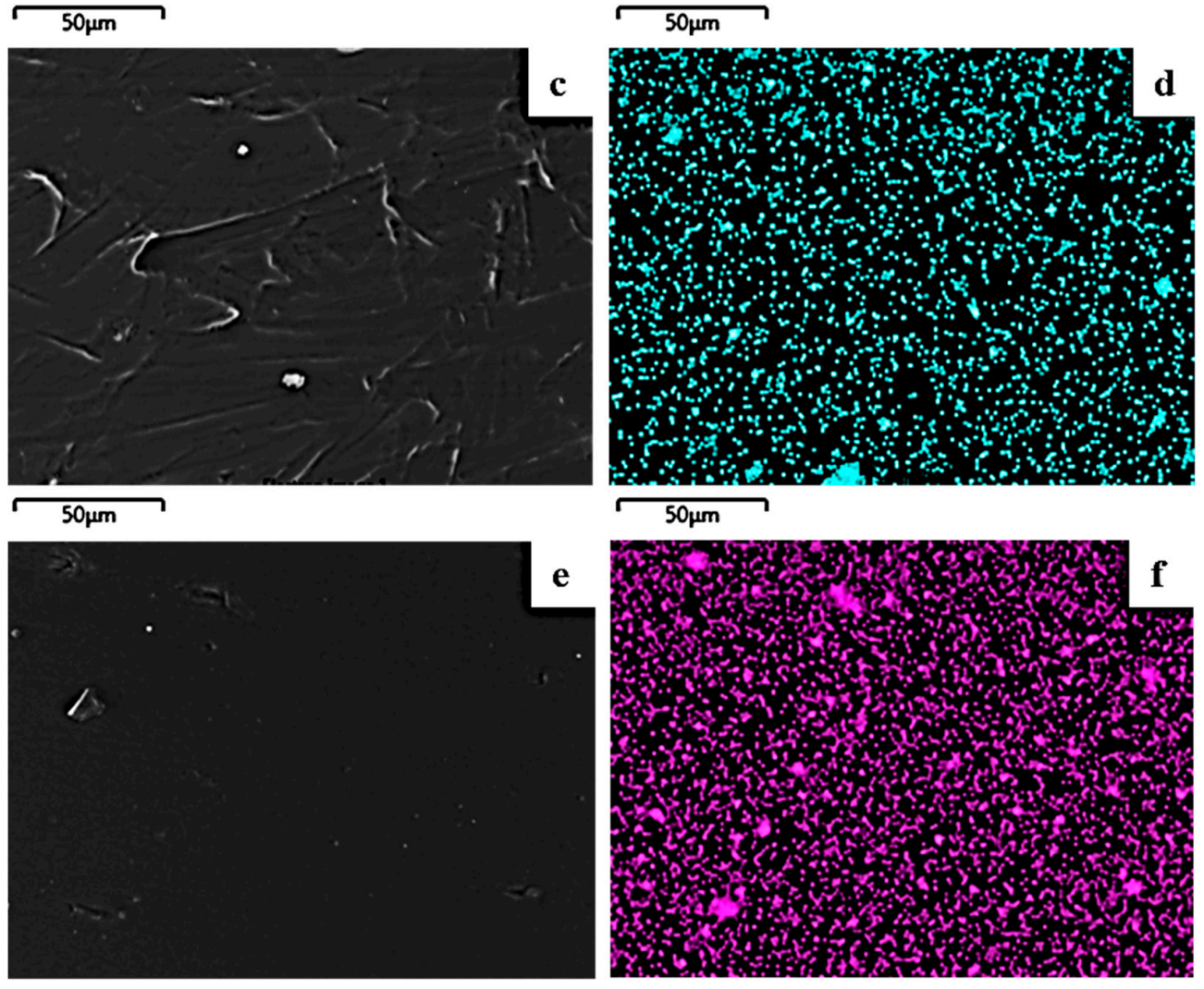

50um

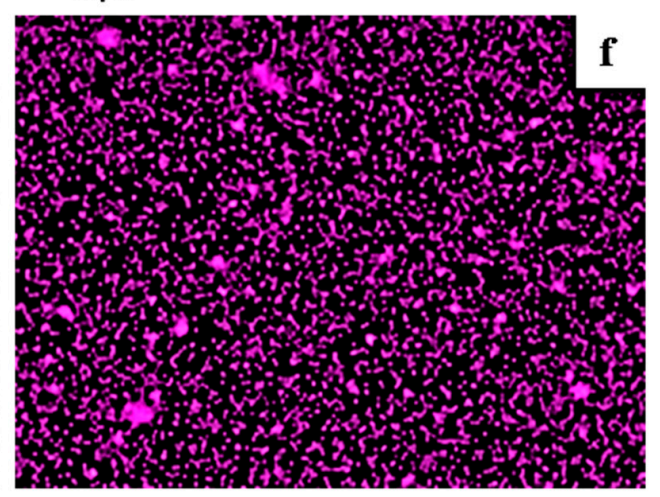

$50 \mu \mathrm{m}$

50um

Figure 7. Secondary electron SEM images and respective EDS compositional mapping of silicon for the films: (a,b) PLLA/1\%TO@NaMt, (c,d) PLLA/3\%TO@NaMt, and (e,f) PLLA/5\%TO@NaMt.

\subsection{Barrier Properties}

Table 3 lists the measured Water Vapor Transmission Rate (WVTR) and Oxygen Transmission Rate (OTR) values as well as the calculated water vapor diffusion coefficient $\left(D_{W V}\right)$ and oxygen permeability coefficient $\left(\mathrm{Pe}_{\mathrm{O} 2}\right)$ values. Water vapor diffusion coefficient values $\left(D_{W V}\right)$ and oxygen permeability coefficient values $\left(\mathrm{Pe}_{\mathrm{O} 2}\right)$ were calculated according to Fick law, oxygen permeability through polymers equation, and assumptions described in [28]. According to this literature, we can calculate the water vapor diffusion coefficient $\mathrm{D}\left(\mathrm{cm}^{2} \cdot \mathrm{s}^{-1}\right)$ for every film as follows:

$$
D_{W V}=W V T R \times \frac{\Delta x}{\Delta C}
$$


where WVTR $\left.\left[\mathrm{G} \cdot \mathrm{cm}^{-2} \cdot \mathrm{s}^{-1}\right)\right]$ is the water vapor transmission rate, $\Delta x(\mathrm{~cm})$ is the film thickness, and $\Delta C\left(\mathrm{~g} \cdot \mathrm{cm}^{-3}\right)$ is the humidity concentration gradient in the two opposite sides of the film. Furthermore, we can calculate oxygen permeability through films as follows:

$$
\frac{J}{A}=P e_{\mathrm{O} 2} \times \frac{\Delta C}{\Delta x}
$$

where $\mathrm{J} / \mathrm{A}\left(\mathrm{mol} \cdot \mathrm{cm}^{-2} \cdot \mathrm{s}^{-1}\right)$ is the specific amount of gas pass through the membrane, $P e_{\mathrm{O} 2}$ $\left(\mathrm{cm}^{2} \cdot \mathrm{s}^{-1}\right)$ is the permeability coefficient, $\Delta C\left(\mathrm{~mol} \cdot \mathrm{cm}^{-3} \mathrm{STP}\right)$ is the pressure gradient in the two opposite sides of the membrane, and $\Delta x(\mathrm{~cm})$ is the membrane thickness.
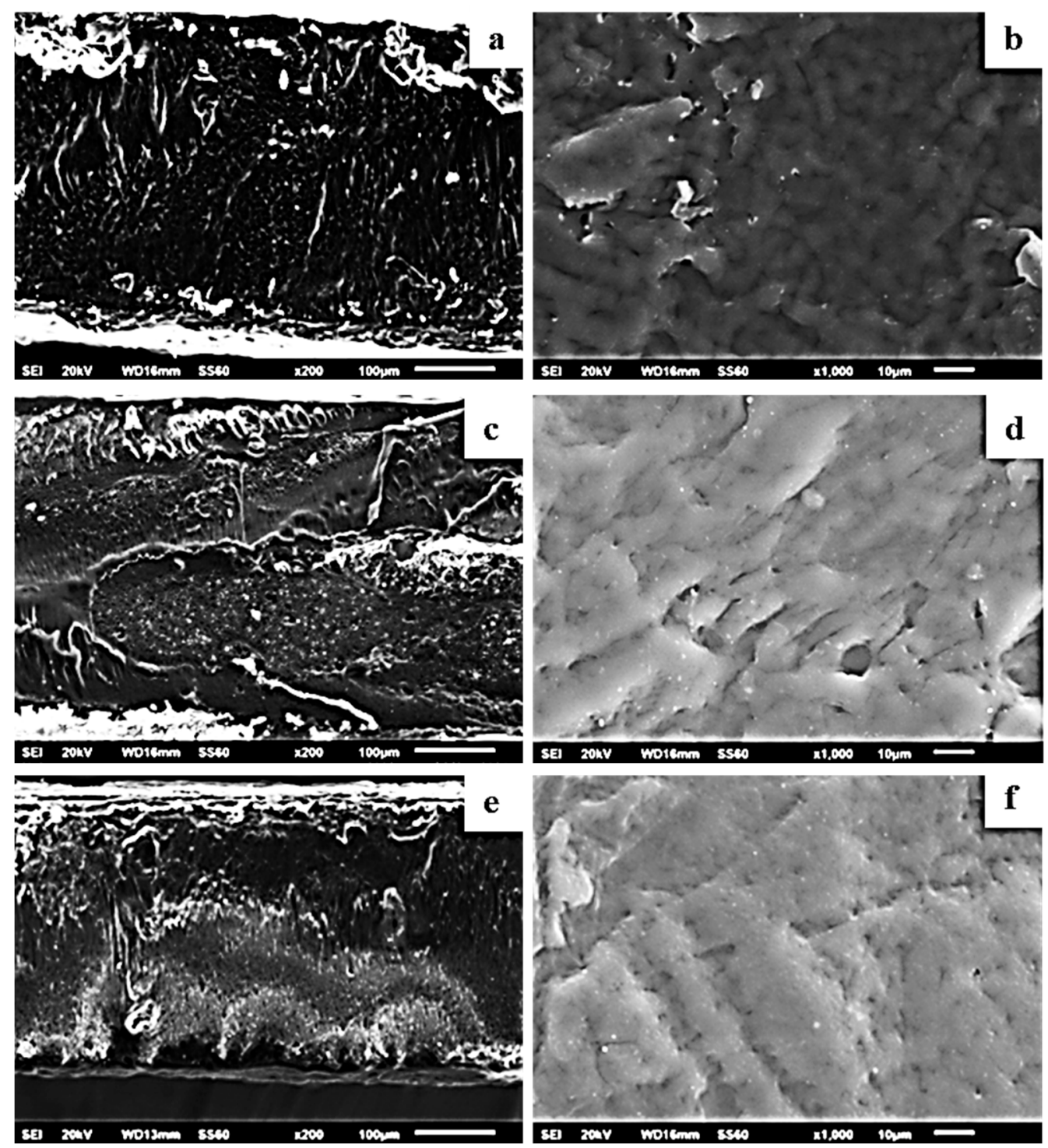

Figure 8. SEM images for the films: (a,b) PLLA/1\%TO@OrgMt, (c,d) PLLA/3\%TO@OrgMt, and (e,f) PLLA/5\%TO@OrgMt. 

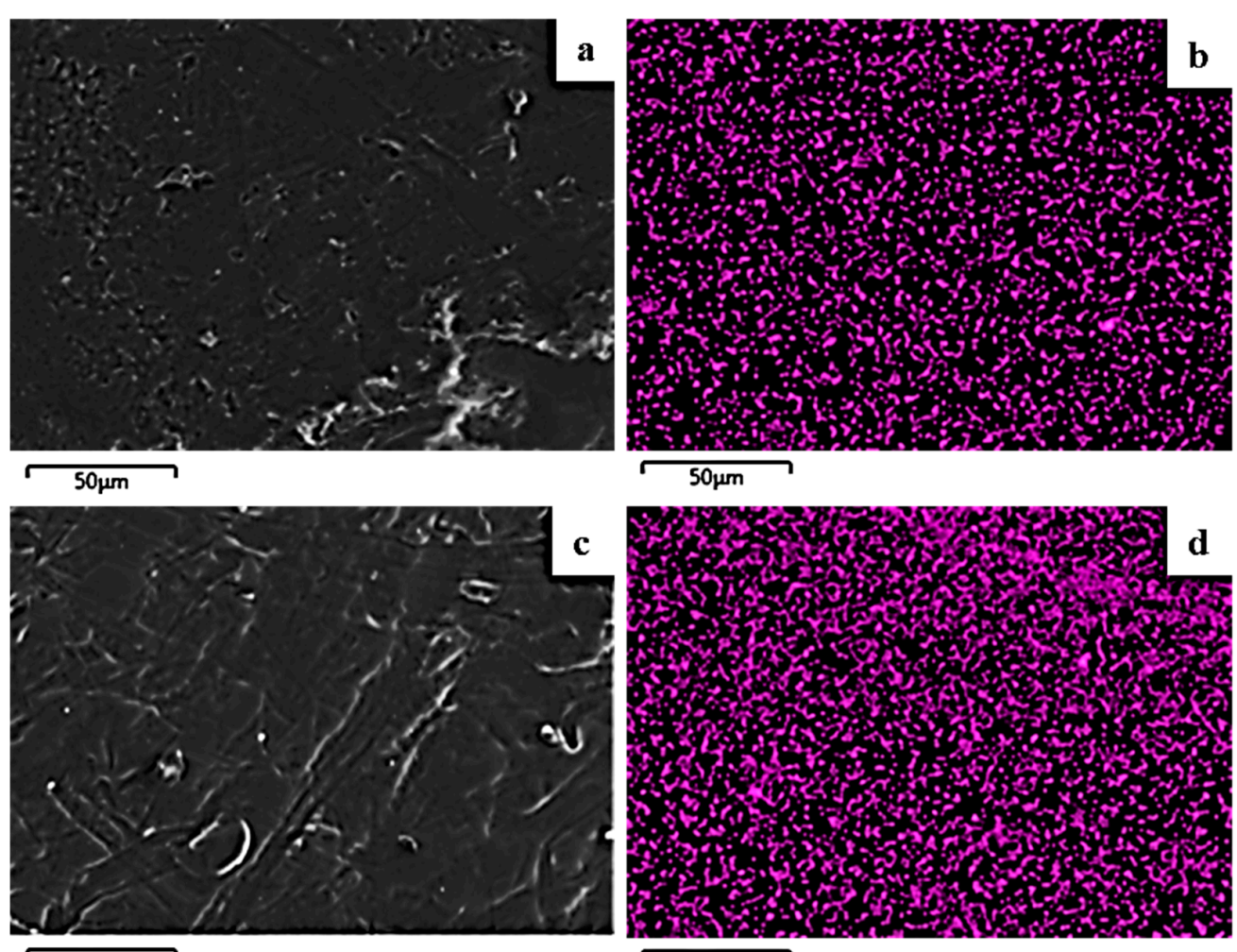

$50 \mu \mathrm{m}$
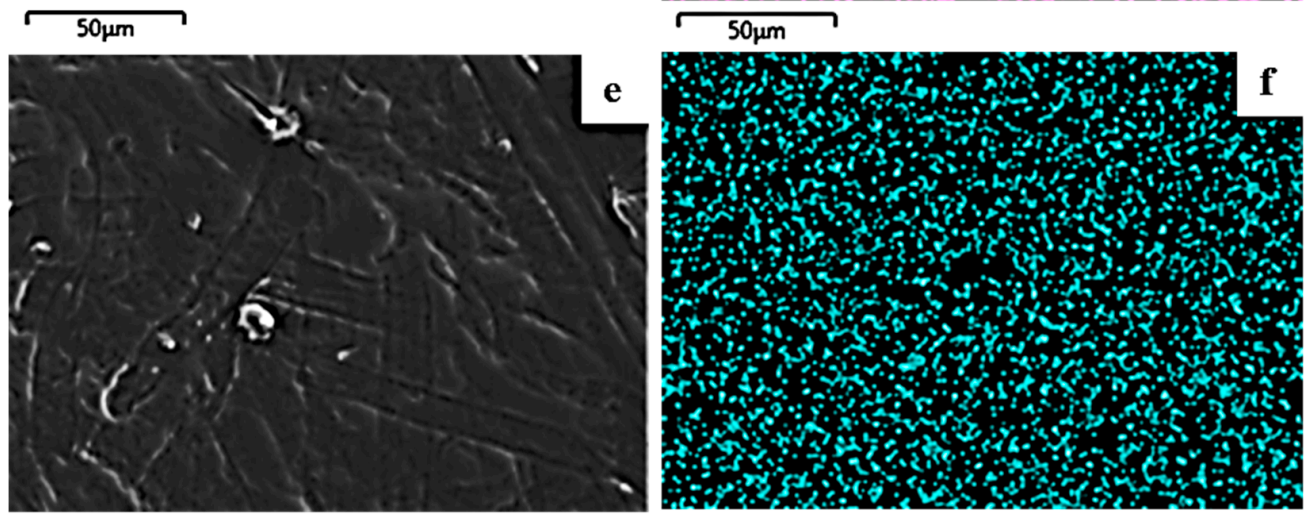

$50 \mu \mathrm{m}$

$50 \mu \mathrm{m}$

Figure 9. Secondary electron SEM images and respective EDS compositional mapping of silicon for the films: (a,b) PLLA/1\%TO@OrgMt, (c,d) PLLA/3\%TO@OrgMt, and (e,f) PLLA/5\%TO@OrgMt.

Rearranging Equation (1), we can express the permeability coefficient as follows:

$$
P e_{\mathrm{O} 2}=\frac{J}{A \times \Delta C} \times \Delta x
$$

Based on these two mass transfer parameters and considering the value of $5 \mathrm{wt} \%$ as the maximum additive concentration in the PLLA matrix, it is obvious from Table 3 that the addition of NaMt clay in the PLLA matrix decreases the water vapor barrier, while the addition of OrgMt clay increases this property. The presence of TO in both types of clay increases the water vapor barrier further. In contrast, the oxygen barrier increases by the addition of either NaMt or OrgMt in the PLLA matrix. Similarly to the water vapor diffusion case, the addition of TO in both types of clay leads to higher oxygen barrier values. In conclusion, we can say that the exfoliated PLLA/NaMt samples exhibit lower water vapor barrier values, even from values of the pure PLLA, compared to the intercalated PLLA/OrgMt samples, which exhibit higher water vapor barrier values from the values of 
both the pure PLLA and the PLLA/NaMt samples. In all cases, the oxygen permeability barrier increases.

Table 3. Water Vapor Transmission Rate (WVTR), Oxygen Transmission Rate (OTR), and antioxidant activity values of all tested PLLA/xclay and PLLA/xTO@clay composite films.

\begin{tabular}{|c|c|c|c|c|c|}
\hline Sample Code Name & $\begin{array}{c}\text { WVTR } \\
\left(\mathrm{g} \cdot \mathrm{m}^{-2} \cdot \mathrm{day}^{-1}\right)\end{array}$ & $\begin{array}{c}D_{W V} \\
\left(\mathrm{~cm}^{2} \cdot \mathrm{s}^{-1}\right)\end{array}$ & $\begin{array}{c}\text { OTR } \\
\left(\mathrm{cm}^{3} \cdot \mathrm{m}^{-2} \cdot \mathrm{day}^{-1}\right)\end{array}$ & $\begin{array}{c}\mathrm{Pe}_{\mathrm{O} 2} \\
\left(\mathrm{~cm}^{2} \cdot \mathrm{s}^{-1}\right)\end{array}$ & $\begin{array}{c}\text { Antioxidant } \\
\text { Activity after } 24 \mathrm{~h}\end{array}$ \\
\hline PLLA & $15.9 \pm 1.3$ & $9.64( \pm 0.9) \times 10^{-12}$ & $12.4 \pm 0.9$ & $5.39( \pm 0.6) \times 10^{-10}$ & n.d. ${ }^{1}$ \\
\hline PLLA/1NaMt & $16.1 \pm 1.2$ & $9.76( \pm 0.8) \times 10^{-12}$ & $9.8 \pm 0.8$ & $4.26( \pm 0.5) \times 10^{-10}$ & n.d. \\
\hline PLLA/3NaMt & $16.4 \pm 1.4$ & $9.96( \pm 1.1) \times 10^{-12}$ & $9.4 \pm 0.8$ & $4.06( \pm 0.5) \times 10^{-10}$ & n.d. \\
\hline PLLA/5NaMt & $16.7 \pm 1.0$ & $10.14( \pm 0.7) \times 10^{-12}$ & $9.2 \pm 0.7$ & $4.00( \pm 0.4) \times 10^{-10}$ & n.d. \\
\hline PLLA/1TO@NaMt & $15.0 \pm 1.1$ & $9.11( \pm 0.8) \times 10^{-12}$ & $9.0 \pm 0.6$ & $3.91( \pm 0.3) \times 10^{-10}$ & $11.9 \pm 1.2$ \\
\hline PLLA/3TO@NaMt & $15.1 \pm 1.2$ & $9.17( \pm 0.8) \times 10^{-12}$ & $8.9 \pm 0.6$ & $3.88( \pm 0.3) \times 10^{-10}$ & $19.7 \pm 1.3$ \\
\hline PLLA/5TO@NaMt & $15.5 \pm 1.3$ & $9.41( \pm 0.9) \times 10^{-12}$ & $8.8 \pm 0.7$ & $3.84( \pm 0.4) \times 10^{-10}$ & $24.1 \pm 1.5$ \\
\hline PLLA/1OrgMt & $14.9 \pm 0.9$ & $9.03( \pm 0.6) \times 10^{-12}$ & $9.3 \pm 0.8$ & $4.03( \pm 0.5) \times 10^{-10}$ & n.d. \\
\hline PLLA/3OrgMt & $14.1 \pm 0.9$ & $8.54( \pm 0.6) \times 10^{-12}$ & $8.4 \pm 0.7$ & $3.65( \pm 0.4) \times 10^{-10}$ & n.d. \\
\hline PLLA/5OrgMt & $13.6 \pm 0.8$ & $8.26( \pm 0.5) \times 10^{-12}$ & $6.3 \pm 0.5$ & $2.72( \pm 0.2) \times 10^{-10}$ & n.d. \\
\hline PLLA/1TO@OrgMt & $14.0 \pm 1.0$ & $8.50( \pm 0.7) \times 10^{-12}$ & $9.1 \pm 0.6$ & $3.95( \pm 0.3) \times 10^{-10}$ & $17.2 \pm 1.8$ \\
\hline PLLA/3TO@OrgMt & $13.6 \pm 0.8$ & $8.26( \pm 0.5) \times 10^{-12}$ & $8.0 \pm 0.6$ & $3.48( \pm 0.3) \times 10^{-10}$ & $38.0 \pm 2.1$ \\
\hline PLLA/5TO@OrgMt & $13.0 \pm 0.7$ & $7.92( \pm 0.4) \times 10^{-12}$ & $5.3 \pm 0.5$ & $2.30( \pm 0.2) \times 10^{-10}$ & $50.3 \pm 2.3$ \\
\hline
\end{tabular}

\subsection{Antioxidant Activity}

Table 3 reports the antioxidant activity values calculated from Equation (4). All tested films exhibit significant antioxidant activity, which indicates that, potentially, they could be used as active packaging films. It is obvious from measurements that the antioxidant activity increases as the content of TO@NaMt and TO@OrgMt nanostructure increases. Antioxidant activity was calculated by absorbance measurements of DPPH ethanolic solution at $517 \mathrm{~nm}$. The presence of film pieces in such solutions decreases the absorbance values. The following equation estimates the $\%$ antioxidant activity of each sample film:

$$
\% \text { Antioxidant activity }=\left(\mathrm{Abs}_{\mathrm{control}}-\mathrm{Abs}_{\text {sample }}\right) / \mathrm{Abs}_{\text {control }} \times 100
$$

where $\mathrm{Abs}_{\text {control }}$ is the absorbance value of pure DPPH ethanolic solution and $\mathrm{Abs}_{\text {sample }}$ is absorbance value of DPPH ethanolic solution after incubation with $30 \mathrm{mg}$ sample film for $24 \mathrm{~h}$ in a dark place and at $25^{\circ} \mathrm{C}$. TO@OrgMt-based films exhibit higher antioxidant activity compared to that of the TO@NaMt-based films. This result could be attributed to the higher TO content of TO@OrgMt nanostructure (36.2 wt $\%$ ) over that of TO@NaMt nanostructure $(22.0 \mathrm{wt} \%)$.

\subsection{Antimicrobial Properties}

Antibacterial activity of thyme oil and PLLA films was tested against four food pathogenic bacteria, E. coli, S. aureus, S. enterica, and L. monocytogenes. The inhibition zones indicated strong antimicrobial activity of pure thyme oil against the tested bacteria. Thyme oil totally inhibited the E. coli and L. monocytogenes and also exhibited good antibacterial activity against $S$. aureus and $S$. enterica. The mean inhibition zone diameter for the last two microorganisms was $55 \mathrm{~mm}$ and $27 \mathrm{~mm}$, respectively. On the contrary, the tested films did not show antibacterial effect neither in terms of surrounding clear zone nor underneath the film. Many parameters could affect the film's antimicrobial properties, e.g., the nature of the $\mathrm{EO}$, the quantity or the mass ratio of $\mathrm{EO}$ into the polymer, and maybe the combination of some of them. By reviewing the results, it seems that the film formation process by hot-pressing at high temperatures $\left(160^{\circ} \mathrm{C}\right)$ could evaporate the EO's volatile and bioactive compounds, leading to a high decrease of their antibacterial activity. Additionally, this hot-pressing process probably removes the EO from the tested film and reduces drastically the EO's concentration in films, causing a non-antimicrobial effect. Finally, it was reported 
in the literature the possibility that the controlled EO's releasing from the polymer films could limit the diffusion of the EO's active components [29].

\section{Materials and Methods}

\subsection{Materials}

\subsubsection{Used Essential Oil}

Thyme oil used was purchased from Esperis S.p.a., Binda, Milano, Italy, and, according to safety data sheets, consists of 50-60\% thymol; $15-20 \%$ para-cymene; $10-12.5 \%$ 1-isopropyl-4-methyl-1,4-cyclohexadiene p-mentha-1,4-diene; 3-5\% carvacrol; and 1-3\% linalool, beta-caryophyllene, beta-myrcene, (R)-p-mentha-1,8-diene, alpha-pinene, borneol, and terpinene-4-olo.

\subsubsection{Used Clay}

(i) Sodium exchanged montmorillonite (NaMt) with code name Nanomer ${ }^{\circledR}$ PGV with mass density $2.6 \mathrm{~g} / \mathrm{cm}^{3}$ and CEC value $145 \mathrm{meq} / 100 \mathrm{~g}$ was produced by Nanocor Company (Hoffman Estates, IL, USA) and supplied by Aldrich (St. Louis, MO, USA). The chemical composition of $\mathrm{NaMt}$ was $62.9 \% \mathrm{SiO}_{2}, 19.6 \% \mathrm{Al}_{2} \mathrm{O}_{3}, 3.35 \% \mathrm{Fe}_{2} \mathrm{O}_{3}, 3.05 \% \mathrm{MgO}, 1.68 \% \mathrm{CaO}$, and $1.53 \% \mathrm{Na}_{2} \mathrm{O}$. (ii) Organo-montmorillonite (OrgMt) NANOMER ${ }^{\circledR}$-I.44P was produced by Nanocor Company (Hoffman Estates, IL, USA) and supplied by Aldrich (St. Louis, MO, USA). NANOMER ${ }^{\circledR}$-I.44P is an ammonium ion-modified clay containing $\sim 40 \mathrm{wt} \%$ dimethyl dialkyl (C14-18) ammonium organic modifier.

\subsubsection{Used PLLA}

PLLA was supplied by NatureWorks, Minnetonka, MN, USA, with the trade name Ingeo $^{\mathrm{TM}}$ Biopolymer 3052D.

\subsection{Methods}

\subsubsection{Preparation of TO@NaMt and TO@OrgMt Nanostructures}

The encapsulation of TO in both NaMt and OrgMt clays was performed via a green evaporation/adsorption process described in detail previously [20]. The final TO loading on obtained TO@NaMt and TO@OrgMt nanostructures was calculated gravimetrically and was approx. $22.0 \mathrm{wt} \%$ and $36.2 \mathrm{wt} \%$, respectively.

\subsubsection{Preparation of PLLA/TO@NaMt and PLLA/TO@OrgMt Films}

The PLLA/TO@NaMt and PLLA/TO@OrgMt films were prepared via a melt-mixing process. For the preparation, a minilab co-rotating twin extruder (Haake Mini Lab II, ThermoScientific, ANTISEL, S.A., Athens, Greece) was used. The uniform operating temperature was $170{ }^{\circ} \mathrm{C}$ at a screw speed of $100 \mathrm{rpm}$ for $5 \mathrm{~min}$ total processing time. The nominal compositions of TO@NaMt and TO@OrgMt nanohybrids added to PLLA were fixed to $1 \mathrm{wt} \%, 3 \mathrm{wt} \%$, and $5 \mathrm{wt} \%$. "Blank" samples were also prepared for comparison by mixing PLLA with commercial NaMt and OrgMt with the same nominal compositions. The obtained melt compound strands were cut into small granules using a granulating machine. Finally, films were produced with approx. $10 \mathrm{~cm}$ diameter by hot-pressing of approximately $2 \mathrm{~g}$ of the obtained granules at $160^{\circ} \mathrm{C}$ under 3.0 megapascal (MPa) constant pressure for $3 \mathrm{~min}$ using a hydraulic press with heated platens.

\subsection{XRD Analysis}

The crystalline structure of all PLLA/NaMt, PLLA/TO@NaMt, PLLA/OrgMt, and PLLA/TO@OrgMt films was studied via XRD analysis measurements using a Brüker D8 Advance X-ray diffractometer (Brüker, Analytical Instruments, S.A. Athens, Greece) equipped with a LINXEYE XE High-Resolution Energy-Dispersive detector. 


\subsection{FTIR Spectrometry}

The chemical structure and the possible interactions of PLLA chains with NaMt, OrgMt, TO@NaMt, and TO@OrgMt nanostructures of all PLLA/NaMt, PLLA/TO@NaMt, PLLA/OrgMt, and PLLA/TO@OrgMt films were studied by IR spectra measurements. The obtained infrared (FTIR) plots were the average of 32 scans at $2 \mathrm{~cm}^{-1}$ resolution and were measured using an FT/IR-6000 JASCO Fourier transform spectrometer (JASCO, Interlab, S.A., Athens, Greece) in the wavenumber range of $4000-400 \mathrm{~cm}^{-1}$.

\subsection{Thermal Studies TG-DTA}

Thermogravimetric (TGA) and differential thermal analysis (DTA) were carried out on all obtained films. Measurements were performed using a PerkinElmer Pyris Diamond TGA/DTA instrument (Interlab, S.A., Athens, Greece). Samples of approximately $5 \mathrm{mg}$ were heated under $\mathrm{N}_{2}$ flow from 25 to $700{ }^{\circ} \mathrm{C}$ at a rate of $5{ }^{\circ} \mathrm{C} / \mathrm{min}$.

\subsection{Tensile Properties}

Tensile measurements were carried out on all prepared films according to the ASTM D638 method. A Simantzü AX-G 5 kNt instrument (Simantzu. Asteriadis, S.A., Athens, Greece) was used. Three to five samples of each film were tensioned at an across head speed of $2 \mathrm{~mm} / \mathrm{min}$. The samples were dumbbell-shaped with gauge dimensions of $10 \mathrm{~mm} \times 3 \mathrm{~mm} \times 0.22 \mathrm{~mm}$. Force $(\mathrm{N})$ and deformation $(\mathrm{mm})$ were recorded during the test.

\subsection{Scanning Electron Microscopy (SEM)/Energy Dispersive Spectroscopy (EDS)}

Scanning electron microscopy (SEM) images were obtained using a JEOL JSM-6510 LV SEM Microscope (JEOL Ltd., Tokyo, Japan) equipped with an X-Act EDS detector by Oxford Instruments, Abingdon, Oxfordshire, UK (an acceleration voltage of $20 \mathrm{kV}$ was applied) for elemental and mapping analysis. The specimens were sputtered with an Au-Pd thin film (4-8 nm) using a mini sputter coater SC7620 from Quorum Technologies LTD (Kent, UK).

\subsection{Water Vapor Transmission Rate (WVTR)}

WVTR of all obtained films was determined. Experimental conditions were fixed at $38^{\circ} \mathrm{C}$ and 50\% RH according to the ASTM E96/E96M-05 method. Using a handmade apparatus and following the methodology described extensively in a previous publication [30] and modified recently [22,28], WVTR measurements were carried out. For such measurements, film disks of $2.5 \mathrm{~cm}$ diameter and $100 \mu \mathrm{m}$ thickness were used.

\subsection{Oxygen Permeability (OP)}

The oxygen transition rate (OTR) of all obtained films was analyzed using an oxygen permeation analyzer (8001, Systech Illinois Instruments Co., Johnsburg, IL, USA). All samples were tested at $23{ }^{\circ} \mathrm{C}$ and $0 \%$ RH according to the ASTM D 3985 method. OTR values were expressed at $\mathrm{cc}_{2} / \mathrm{m}^{2} /$ day. The average film thickness of the tested samples was in the range of 350 to $400 \mu \mathrm{m}$. The mean OTR value for each kind of film resulted from the measurements of three samples.

\subsection{Antioxidant Activity}

The antioxidant activity of films was evaluated using $300 \mathrm{mg}$ of small pieces (approximately $3 \mathrm{~mm} \times 3 \mathrm{~mm}$ ) of each film. The sample was placed in a dark-colored glass bottle with a plastic screw cap and filled with $10 \mathrm{~mL}$ DPPH ethanolic solution of $50 \mathrm{ppm}(\mathrm{mg} / \mathrm{L})$ concentration. After incubation at $25^{\circ} \mathrm{C}$ for $24 \mathrm{~h}$ in darkness, the \% antioxidant activity values of the films were calculated according to Equation (4). Antioxidant measurements were carried out using a JASCO V530 UV-VIS instrument (JASCO International CO., Ltd., Tokyo, Japan). 


\subsection{Antimicrobial Assay}

PLLA films were tested by agar diffusion method for their efficacy against Gram-negative bacteria Escherichia coli (ATCC 25922) and Salmonella enterica subsp. enterica (DSMZ 17420) and Gram-positive bacteria Staphylococcus aureus (DSMZ 12463) and Listeria monocytogenes (DSMZ 27575). The above bacteria isolates were provided from the Institute of Technology of Agricultural Products, ELGO-DEMETER, Lykovryssi, Greece.

The stock bacteria colonies were cultured overnight in Mueller-Hinton broth to obtain a range of $10^{7}-10^{8} \mathrm{CFU} \mathrm{mL}{ }^{-1}$. Then, a sterile cotton swab was used to inoculate the surface of Mueller-Hinton agar dishes. To achieve homogeneous growth, the plates were rotated every $60^{\circ}$ during the inoculation process.

Films were cut into $6 \mathrm{~mm}$ discs by a circular knife and sterilized using a UV lamp. Film discs were placed on the surface of Mueller-Hinton agar plates, and the diameter of inhibitory zones was measured after $24 \mathrm{~h}$ of incubation at $37^{\circ} \mathrm{C}$. Moreover, the growth of bacteria on the contact area between the films and the agar surface was also evaluated. Thyme oil antibacterial activity was examined by the agar well diffusion method. The EO was cast into Mueller-Hinton agar wells with $6 \mathrm{~mm}$ diameter, and the clear zone of inhibition was recorded. The experiment was repeated three times.

\subsection{Statistical Analysis}

According to the literature, it is usual in such studies to measure every property at least three times under the same conditions to achieve accurate mean values standard deviation values and standard error. Moreover, it is usual to test the inequality of mean values between all kind of samples to promote the better material which exhibits superior properties. All the experimental data for $\mathrm{E}, \sigma_{\mathrm{uts}}, \varepsilon_{\mathrm{b}}(\%), \mathrm{WVTR}, \%$ water sorption, OTR, E. coli, S. aureus, S. enterica, and L. monocytogenes were statistically treated using the statistical software SPSS ver. 20. Statistical tests were carried the way described in previous literature reports [28].

\section{Conclusions}

Characterization techniques indicated that the interplay and compatibility between PLLA chains and clay platelets was more intense in the case of the intercalated OrgMt clay compared to that of the partially exfoliated NaMt clay. This also means a better mixing of these two materials, i.e., PLLA and OrgMt. Furthermore, in both cases of clay addition, the presence of TO boosted the mixing process. The thermal stability of the PLLA matrix was improved by addition of either NaMt or OrgMt clay. In the case of the OrgMt clay, the increase of such stability was higher. Nevertheless, there was an optimum addition around $3 \mathrm{wt} \%$ for optimum mechanical properties. The exfoliated PLLA/NaMt samples exhibited lower water vapor barrier values, even from values of the pure PLLA, compared to the intercalated PLLA/OrgMt samples, which exhibited higher water vapor barrier values from the values of both the pure PLLA and the PLLA/NaMt samples. In all cases, the oxygen barrier increased, and the TO addition decreased, water vapor diffusion and oxygen permeability. Higher TO adsorption capacity of OrgMt-based materials led to higher antioxidant activity. As an overall validation of the materials in our study, all samples could be potentially used for active food packaging applications, but the PLLA/5\%TO@OrgMt film seems to be the most advantageous.

Author Contributions: The synthesis experiments design, A.E.G., C.E.S. and C.P.; Characterization measurements carrying out and interpretation, A.E.G., C.E.S., D.M., A.K. and M.B.; Paper writing, A.E.G., C.E.S., E.K. and C.P.; The overall evaluation of this work, A.E.G., C.E.S. and A.L.; Experimental data analysis and interpretation, A.E.G., C.E.S., A.K, A.A. and C.P.; The XRD, FTIR, OTR, tensile measurements, antioxidant activity, and WVTR experimental measurements carrying out, A.E.G., C.E.S., D.M., A.A. and A.L.; SEM experiment carrying out D.M. and M.B.; Antimicrobial activity tests carrying out E.K. and V.T. All authors have read and agreed to the published version of the manuscript. 
Funding: This research was funded by University of Patras, Greece, the funding program was "MEDICUS", Project F.K. 81541.

Data Availability Statement: The datasets generated for this study are available on request to the corresponding author.

Acknowledgments: The authors would like to thank: (1) the Department of Business Administration of Food and Agricultural Enterprises, University of Patras, Greece, for the access to the XRD, OTR, and Tensile equipment of the Food Technology Laboratory, and (2) the Professor Christoforos A. Krontiras for his support on the grant of PLLA pellets and the fruitful discussions.

Conflicts of Interest: The authors declare no conflict of interest.

Sample Availability: Samples of the compounds are available from the authors.

\section{References}

1. Gross, R.A.; Kalra, B. Biodegradable Polymers for the Environment. Science 2002, 297, 803-807. [CrossRef] [PubMed]

2. Armentano, I.; Bitinis, N.; Fortunati, E.; Mattioli, S.; Rescignano, N.; Verdejo, R.; Lopez-Manchado, M.A.; Kenny, J.M. Multifunctional Nanostructured PLA Materials for Packaging and Tissue Engineering. Prog. Polym. Sci. 2013, 38, 1720-1747. [CrossRef]

3. Arrieta, M.P.; López, J.; Ferrándiz, S.; Peltzer, M.A. Characterization of PLA-Limonene Blends for Food Packaging Applications. Polym. Test. 2013, 32, 760-768. [CrossRef]

4. Gerometta, M.; Rocca-Smith, J.R.; Domenek, S.; Karbowiak, T. Physical and Chemical Stability of PLA in Food Packaging. In Reference Module in Food Science; Elsevier: Amsterdam, The Netherlands, 2019; ISBN 978-0-08-100596-5.

5. Heydari-Majd, M.; Ghanbarzadeh, B.; Shahidi-Noghabi, M.; Najafi, M.A.; Hosseini, M. A New Active Nanocomposite Film Based on PLA/ZnO Nanoparticle/Essential Oils for the Preservation of Refrigerated Otolithes Ruber Fillets. Food Packag. Shelf Life 2019, 19, 94-103. [CrossRef]

6. Villegas, C.; Arrieta, M.P.; Rojas, A.; Torres, A.; Faba, S.; Toledo, M.J.; Gutierrez, M.A.; Zavalla, E.; Romero, J.; Galotto, M.J.; et al. PLA/Organoclay Bionanocomposites Impregnated with Thymol and Cinnamaldehyde by Supercritical Impregnation for Active and Sustainable Food Packaging. Compos. Part B Eng. 2019, 176, 107336. [CrossRef]

7. $\quad$ Bajpai, V.K.; Kamle, M.; Shukla, S.; Mahato, D.K.; Chandra, P.; Hwang, S.K.; Kumar, P.; Huh, Y.S.; Han, Y.-K. Prospects of Using Nanotechnology for Food Preservation, Safety, and Security. J. Food Drug Anal. 2018, 26, 1201-1214. [CrossRef]

8. Giannakas, A.E.; Leontiou, A.A. Montmorillonite Composite Materials and Food Packaging. In Composites Materials for Food Packaging; John Wiley \& Sons, Ltd.: Hoboken, NJ, USA, 2018; pp. 1-71. ISBN 978-1-119-16024-3.

9. Idumah, C.I.; Zurina, M.; Ogbu, J.; Ndem, J.U.; Igba, E.C. A Review on Innovations in Polymeric Nanocomposite Packaging Materials and Electrical Sensors for Food and Agriculture. Compos. Interfaces 2020, 27, 1-72. [CrossRef]

10. Campos-Requena, V.H.; Rivas, B.L.; Pérez, M.A.; Garrido-Miranda, K.A.; Pereira, E.D. Polymer/Clay Nanocomposite Films as Active Packaging Material: Modeling of Antimicrobial Release. Eur. Polym. J. 2015, 71, 461-475. [CrossRef]

11. Busolo, M.A.; Lagaron, J.M. Antioxidant Polyethylene Films Based on a Resveratrol Containing Clay of Interest in Food Packaging Applications. Food Packag. Shelf Life 2015, 6, 30-41. [CrossRef]

12. Giannakas, A. Na-Montmorillonite Vs. Organically Modified Montmorillonite as Essential Oil Nanocarriers for Melt-Extruded Low-Density Poly-Ethylene Nanocomposite Active Packaging Films with a Controllable and Long-Life Antioxidant Activity. Nanomaterials 2020, 10, 1027. [CrossRef]

13. Shemesh, R.; Krepker, M.; Natan, M.; Danin-Poleg, Y.; Banin, E.; Kashi, Y.; Nitzan, N.; Vaxman, A.; Segal, E. Novel LDPE/Halloysite Nanotube Films with Sustained Carvacrol Release for Broad-Spectrum Antimicrobial Activity. RSC Adv. 2015, 5, 87108-87117. [CrossRef]

14. de Oliveira, L.H.; Trigueiro, P.; Souza, J.S.N.; de Carvalho, M.S.; Osajima, J.A.; da Silva-Filho, E.C.; Fonseca, M.G. Montmorillonite with Essential Oils as Antimicrobial Agents, Packaging, Repellents, and Insecticides: An Overview. Colloids Surf. B Biointerfaces 2022, 209, 112186. [CrossRef] [PubMed]

15. Cheikh, D.; Majdoub, H.; Darder, M. An Overview of Clay-Polymer Nanocomposites Containing Bioactive Compounds for Food Packaging Applications. Appl. Clay Sci. 2022, 216, 106335. [CrossRef]

16. Dainelli, D.; Gontard, N.; Spyropoulos, D.; Zondervan-van den Beuken, E.; Tobback, P. Active and Intelligent Food Packaging: Legal Aspects and Safety Concerns. Trends Food Sci. Technol. 2008, 19, S103-S112. [CrossRef]

17. Rhim, J.-W.; Hong, S.-I.; Ha, C.-S. Tensile, Water Vapor Barrier and Antimicrobial Properties of PLA/Nanoclay Composite Films. LWT-Food Sci. Technol. 2009, 42, 612-617. [CrossRef]

18. Ramos, M.; Fortunati, E.; Beltrán, A.; Peltzer, M.; Cristofaro, F.; Visai, L.; Valente, A.J.M.; Jiménez, A.; Kenny, J.M.; Garrigós, M.C. Controlled Release, Disintegration, Antioxidant, and Antimicrobial Properties of Poly (Lactic Acid)/Thymol/Nanoclay Composites. Polymers 2020, 12, 1878. [CrossRef]

19. Oliver-Ortega, H.; Tresserras, J.; Julian, F.; Alcalà, M.; Bala, A.; Espinach, F.X.; Méndez, J.A. Nanocomposites Materials of PLA Reinforced with Nanoclays Using a Masterbatch Technology: A Study of the Mechanical Performance and Its Sustainability. Polymers 2021, 13, 2133. [CrossRef] 
20. Giannakas, A.; Tsagkalias, I.; Achilias, D.S.; Ladavos, A. A Novel Method for the Preparation of Inorganic and Organo-Modified Montmorillonite Essential Oil Hybrids. Appl. Clay Sci. 2017, 146, 362-370. [CrossRef]

21. Giannakas, A.; Stathopoulou, P.; Tsiamis, G.; Salmas, C. The Effect of Different Preparation Methods on the Development of Chitosan/Thyme Oil/Montmorillonite Nanocomposite Active Packaging Films. J. Food Proc. Preserv. 2019, 44, e14327. [CrossRef]

22. Giannakas, A.E.; Salmas, C.E.; Karydis-Messinis, A.; Moschovas, D.; Kollia, E.; Tsigkou, V.; Proestos, C.; Avgeropoulos, A.; Zafeiropoulos, N.E. Nanoclay and Polystyrene Type Efficiency on the Development of Polystyrene/Montmorillonite/Oregano Oil Antioxidant Active Packaging Nanocomposite Films. Appl. Sci. 2021, 11, 9364. [CrossRef]

23. Wasanasuk, K.; Tashiro, K.; Hanesaka, M.; Ohhara, T.; Kurihara, K.; Kuroki, R.; Tamada, T.; Ozeki, T.; Kanamoto, T. Crystal Structure Analysis of Poly(l-Lactic Acid) $\alpha$ Form On the Basis of the 2-Dimensional Wide-Angle Synchrotron X-Ray and Neutron Diffraction Measurements. Macromolecules 2011, 44, 6441-6452. [CrossRef]

24. Hosen, M.S.; Rahaman, M.H.; Gafur, M.A.; Habib, R.; Qadir, M.R. Preparation and Characterization of Poly(L-Lactic Acid)/Chitosan/Microcrystalline Cellulose Blends. Chem. Sci. Int. J. 2017, 21, 1-10. [CrossRef]

25. Duarte, A.R.C.; Mano, J.F.; Reis, R.L. Novel 3D Scaffolds of Chitosan-PLLA Blends for Tissue Engineering Applications: Preparation and Characterization. J. Supercrit. Fluids 2010, 54, 282-289. [CrossRef]

26. Krishna, S.V.; Pugazhenthi, G. Properties and Thermal Degradation Kinetics of Polystyrene/Organoclay Nanocomposites Synthesized by Solvent Blending Method: Effect of Processing Conditions and Organoclay Loading. J. Appl. Polym. Sci. 2011, 120, 1322-1336. [CrossRef]

27. Pugazhenthi, G.; Suresh, K.; Vinoth Kumar, R.; Kumar, M.; Rajkumar Surin, R. A Simple Sonication Assisted Solvent Blending Route for Fabrication of Exfoliated Polystyrene (PS)/Clay Nanocomposites: Role of Various Clay Modifiers. Mater. Today Proc. 2018, 5, 13191-13210. [CrossRef]

28. Salmas, C.E.; Giannakas, A.E.; Baikousi, M.; Kollia, E.; Tsigkou, V.; Proestos, C. Effect of Copper and Titanium-Exchanged Montmorillonite Nanostructures on the Packaging Performance of Chitosan/Poly-Vinyl-Alcohol-Based Active Packaging Nanocomposite Films. Foods 2021, 10, 3038. [CrossRef]

29. Qin, Y.; Li, W.; Liu, D.; Yuan, M.; Li, L. Development of Active Packaging Film Made from Poly (Lactic Acid) Incorporated Essential Oil. Prog. Org. Coat. 2017, 103, 76-82. [CrossRef]

30. Giannakas, A.; Spanos, C.G.; Kourkoumelis, N.; Vaimakis, T.; Ladavos, A. Preparation, Characterization and Water Barrier Properties of PS/Organo-Montmorillonite Nanocomposites. Eur. Polym. J. 2008, 44, 3915-3921. [CrossRef] 